\title{
Universality in complex networks: random matrix analysis
}

\author{
Jayendra N. Bandyopadhyay ${ }^{1}$ and Sarika Jalan ${ }^{2}$ \\ ${ }^{1}$ Max-Planck Institute for the Physics of Complex Systems, Nöthnitzerstr. 38, D-01187 Dresden, Germany \\ ${ }^{2}$ Max-Planck Institute for Mathematics in the Sciences, Inselstrasse 22, D-04103 Leipzig, Germany
}

\begin{abstract}
We apply random matrix theory to complex networks. We show that nearest neighbor spacing distribution of the eigenvalues of the adjacency matrices of various model networks, namely scalefree, small-world and random networks follow universal Gaussian orthogonal ensemble statistics of random matrix theory. Secondly we show an analogy between the onset of small-world behavior, quantified by the structural properties of networks, and the transition from Poisson to Gaussian orthogonal ensemble statistics, quantified by Brody parameter characterizing a spectral property. We also present our analysis for a protein-protein interaction network in budding yeast.

PACS numbers: 89.75.Hc,64.60.Cn,89.20.-a
\end{abstract}

The network concept has been gaining recognition as a fundamental tool in understanding dynamical behavior and response of real systems coming from different fields such as biology (e.g. food-web, nervous system, cellular metabolism, protein-protein interaction network, gene regulatory networks), social systems (e.g. scientific collaboration, citation), linguistic networks, and technological systems (e.g. Internet, power-grid etc (for reviews, see, e.g., [1]).

Different models have been proposed to study and understand systems having underlying network structures. Watts and Strogatz proposed an algorithm to generate popularly known as 'small-world network' 2], which captures randomness (characterized by small diameter) and regularity (measured by clustering) of real-world networks. This model emphasizes on the importance of random connections in networks. Barabási and Albert proposed a model to capture degree distributions of realworld networks [3]. According to this model only few nodes are responsible to carry the whole network. Since then came spurt of activities to the network studies and various structural properties of these model networks and real world networks have been studied to a great extent $[1,2,3,4]$.

Furthermore, there exists extensive literature demonstrating that the properties of networks are well characterized by the spectrum of associated adjacency matrices. The adjacency matrix $(A)$ of a network is defined in the following way: $A_{i j}=1$ if $i$ and $j$ nodes are connected and zero otherwise. For an undirected network it is symmetric and consequently has real eigenvalues. These eigenvalues give information about some basic topological properties of underlying networks [5]. For example, spectral density of adjacency matrix of a random network, whose elements are randomly 0 or 1 , also follows the semicircular law [6]. Interestingly, this result matches with a very celebrated result in RMT about the spectral density of a random matrix, whose elements are Gaussian distributed random numbers, following Wigner's semicircular law [7].

With the increasing availability of large maps of realworld networks, the analysis of spectral densities of realworld networks and model networks having real-world properties have also begun [6, [8, 9]. These analyses show that the spectral densities of model networks and realworld networks are not semicircular, instead they have some specific features depending on the minute details of the networks . For example, small-world model networks show very complex spectral densities with many sharp peaks, while spectral densities of scale-free model networks exhibit triangular distribution [6, 9].

In this paper we study networks within the framework of random matrix theory (RMT). We show that there exists one to one correlation between the network diameter which is a structural property and the eigenvalues fluctuations of the adjacency matrix which is a spectral property. We present our RMT analysis for various model networks studied extensively in the recent network literature and also for a real-world network. We find that in spite of having differences (in terms of various local and global properties, which are being used to characterize networks) in these networks, fluctuations of the eigenvalues of adjacency matrices show universal distribution. So far we are aware of only one relevant paper where authors have studied eigenvalue fluctuations in a microarray data for discovering functional gene modules [10].

RMT was proposed by Wigner to explain statistical properties of nuclear spectra [7]. Later this theory was successfully applied in the study of spectra of different complex systems including disordered systems, quantum chaotic systems, large complex atoms, etc 11]. More recently, RMT is applied successfully to analyze timeseries data of stock-market, atmosphere, human EEG, and many more [12]. A popular practice in RMT is to study eigenvalue fluctuations via nearest neighbor spacing distribution (NNSD). NNSD is the distribution of spacings between consecutive eigenvalues. It follows two universal properties depending upon the underlying correlations among the eigenvalues. For correlated eigenvalues, NNSD follows Wigner-Dyson formula of Gaussian orthogonal ensemble (GOE) statistics of RMT, which is a property shown by real symmetric random matrices with elements being Gaussian distributed random numbers. On the other hand, for uncorrelated eigenvalues, NNSD follows Poisson statistics of RMT, which is a property shown by random matrices having nonzero elements only along its diagonals. 

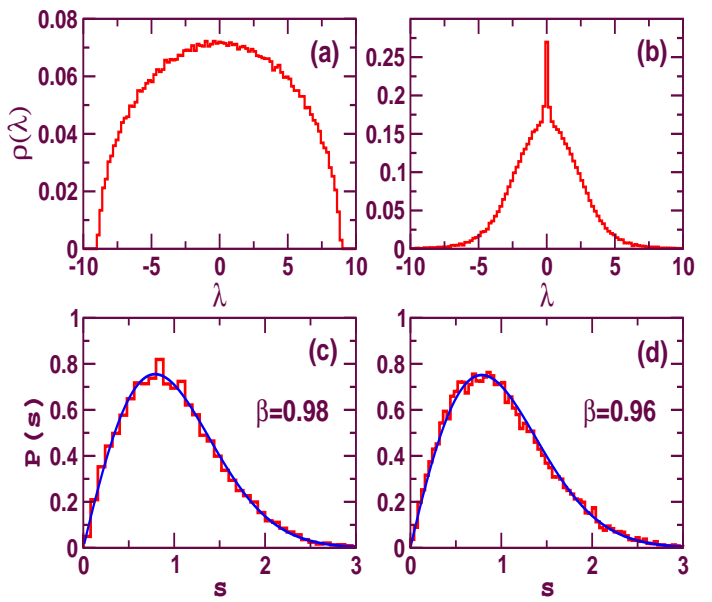

FIG. 1: (Color online) (a)-(b) Spectral density $(\rho(\lambda))$ of random (Erdös-Renyi model) and scale-free network (following Ref.[3]), respectively. (c)-(d) Corresponding spacing distribution $(P(s))$. Both follow GOE statistics. The histograms are numerical results and the solid lines represent fitted Brody distribution. All networks have $N=2000$ nodes and an average degree $k=20$ per node. Figures are plotted for average over 10 random realizations of the networks.

In the present study, we find that the NNSD of random networks follow GOE. The spectral density of random networks and of Gaussian distributed random matrices are both semicircular, so it was expected that their spacing distributions would be identical. However, very interestingly, NNSD of scale-free networks and small-world networks also follow GOE statistics. In addition to these model networks, we also analyze a protein-protein interaction network in budding yeast. We find that this realworld network is scale-free and its spacing distribution also follows GOE.

Secondly, we study the change of NNSD with the transition from regular to small-world network. WattsStrogatz model of small-world network is constructed by rewiring the edges of a regular ring lattice with probability $p$. This rewiring procedure generates a network with some random connections, without altering the number of vertices or edges. For $p=0$, structure of the regular lattice or $k$-nearest neighbor coupled network remains same; on the other hand, for $p=1$, the regular lattice becomes a random network. For intermediate values of $p$, the graph is a small-world network. We find that for the regular lattice $(p=0)$, NNSD follows Poisson statistics, for $p=1$ it follows GOE statistics and for $0<p<1$ it shows intermediate statistics of Poisson and GOE. Moreover we show that the NNSD changes from Poisson to GOE with a very small increment in $p$, and most importantly, transition to GOE takes place exactly at the onset of small-world transition. We establish a relation between small-world transition and GOE transition by comparing the diameter and the clustering coefficients of network with the Brody parameter $\beta$. This parameter comes from a semiempirical eigenvalues spacing distribu- tions studied extensively in RMT to model Poisson to GOE transition.

Here we briefly describe some aspects of RMT which we use in our network analysis. We denote the eigenvalues of a network by $\lambda_{i}, i=1, \ldots, N$, where $N$ is size of the network and $\lambda_{1}<\lambda_{2}<\lambda_{3}<\cdots<\lambda_{N}$. In order to get universal properties of the fluctuations of eigenvalues, it is customary in RMT to unfold the eigenvalues by a transformation $\bar{\lambda}_{i}=\bar{N}\left(\lambda_{i}\right)$, where $\bar{N}$ is averaged integrated eigenvalue density [7]. Since we do not have any analytical form for $\bar{N}$, we numerically unfold the spectrum by polynomial curve fitting (for elaborate discussion on unfolding, see Ref.[7]). After unfolding, average spacings will be unity, independent of the system. Using the unfolded spectra, we calculate spacings as $s_{i}=\bar{\lambda}_{i+1}-\bar{\lambda}_{i}$. NNSD is defined as the probability distribution $(P(s))$ of these $s_{i}$ 's. In case of Poisson statistics, $P(s)=\exp (-s)$; whereas for GOE, $P(s)=\frac{\pi}{2} s \exp \left(-\frac{\pi s^{2}}{4}\right)$. For intermediate cases, the spacing distribution is described by Brody distribution [13]:

$$
P_{\beta}(s)=A s^{\beta} \exp \left(-\alpha s^{\beta+1}\right),
$$

where

$$
A=(1+\beta) \alpha \text { and } \alpha=\left[\Gamma\left(\frac{\beta+2}{\beta+1}\right)\right]^{\beta+1}
$$

This is a semiempirical formula characterized by parameter $\beta$. As $\beta$ goes from 0 to 1 , the Brody distribution smoothly changes from Poisson to GOE. We fit spacing distributions of different networks by the Brody distribution $P_{\beta}(s)$. This fitting gives an estimation of $\beta$, and consequently identifies whether the spacing distribution of a given network is Poisson, GOE or intermediate of these two.

In Fig. 1, we present the ensemble averaged spectral density $(\rho(\lambda))$ and spacing distribution $(P(s))$ of random and the scale-free networks. Figs. 1(a) and 1(b) respectively show the well known semicircular and triangular distribution of spectral densities of random and scale-free networks. Using RMT techniques described earlier we obtain spacing distributions for the unfolded eigenvalues. Figs. 1(c) and 1(d) plot these distributions. Now using Eq. (1), we estimate Brody parameter as $\beta \simeq 1$, which clearly shows GOE statistics of spacing distributions for both the networks. Following RMT, these results imply that even though spectral densities of scale-free networks are different from random networks, correlations among the eigenvalues of scale-free networks are as strong as that of the random networks.

To show that our analysis exhibiting universality of GOE statistics for model random networks are generic, we studied some real-world networks also and here we present our results for a protein-protein interaction network in budding yeast [14]. Results are presented in Fig. 22 top panel showing that the degree distribution $p(k)$ of the network follows power-law, i.e., $p(k) \propto k^{-\gamma}$, with $\gamma \simeq 2.1536$. Middle panel shows that the spectral 

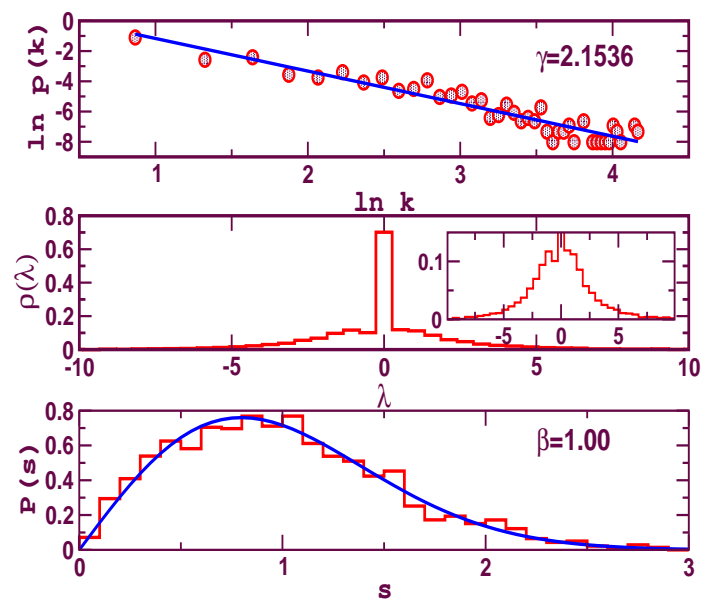

FIG. 2: (Color online) Figure shows different properties of a protein-protein interaction network in budding yeast. (a) Degree distribution : the scale-free nature of the network is clearly observed. (b) Spectral density : large value of $\rho(0)$ (Inset : besides large $\rho(0)$, overall spectral density follows well-known triangular distribution. (c) Spacing distribution : it follows GOE, estimated value of $\beta$ is $\sim 1$. The histogram represents numerical result and the solid line is fitted Brody distribution given by Eq. (1).

density of this network is overall triangular (see also the inset of this panel for magnified figure) but with very large $\rho(0)$. Large value of $\rho(0)$ is one of the characteristics of many real-world networks [9]. Due to the large $\rho(0)$, it is very difficult to numerically unfold the spectra. Therefore, in this case, we divide the spectra into two parts : one part contains only negative eigenvalues with values less than -0.1 and the other part contains positive eigenvalues with values greater than 0.1 . We assume these two sets of eigenvalues as an ensemble of two realizations, and calculate ensemble averaged spacing distribution. Bottom panel of Fig. 2 is showing that the spacing distribution of this protein-protein interaction network follows GOE. We have also studied spectral rigidity of the eigenvalues spectra which show that these networks follow RMT predictions for sufficiently large scales. Also this analysis seems to characterize the level of randomness in network architecture [15].

Now we discuss our results for the Watts-Strogatz model of small-world network. In Figs. 3(a) and 3(e), we present respectively the spectral density and the spacing distribution of regular ring lattice with each node having 20 edges. Spacing distributions are obtained again from the same technique. Subfigure (a) shows that the spectral density of lattice is complicated without having any known analytical form; but its spacing distribution (subfigure (e)) clearly follows Poisson statistics $(\beta \sim 0)$. Then we randomize a fraction $p=5 \times 10^{-5}$ of the edges of regular lattice. For this value of $p$, spectral density and spacing distribution are plotted respectively in Fig. B(b) and Fig. 3(f). These figures reveal that, for this very small value of $p$, spectral density does not show any notice- able change as compared to the regular lattice, whereas spacing distribution shows different property $(\beta \sim 0.08)$. As we further increase parameter $p$ from $5 \times 10^{-5}$ to $p=2 \times 10^{-4}$ and thereafter to $p=5 \times 10^{-4}$, spectral densities show hardly any changes in its features (Figs. 3(c)-3(d)), but very interestingly, according to Figs. 3(g) and $3(\mathrm{~h})$, spacing distributions show significantly different properties as compared to the regular lattice. Now these are looking like intermediate of the Poisson and the GOE. By fitting spacing distribution corresponding to these two $p$ values with the Brody formula (Eq. 11), we estimate $\beta$ respectively as 0.63 and 0.79 . These values indicate that we are already at the onset of Poisson $\rightarrow \mathrm{GOE}$ transition. Note that we take regular lattice with average degree $k \simeq 20$ for which NNSD is showing Poisson statistics. For other values of $k$, where we may not have Poisson statistics, there also we get transition to GOE statistics. We choose $k \simeq 20$ just to make transition to GOE analogy clear. Detailed analysis for other $k$ values would be presented elsewhere [16].

We present in Fig. 4 variation of $\beta$ as a function of $p$ over the whole range $0 \leq p \leq 1$. Here we show correspondence between the Brody parameter and two important network parameters - the characteristic path length $L(p)$ and the clustering coefficient $C(p)$ - as a function of $p$. $L$ measures number of connections in the shortest path between two nodes, averaged over all pairs of the nodes. Clustering coefficient $C$ measures the cliquishness of a typical neighborhood, averaged over all nodes. In this figure we have normalized $L$ and $C$ by values $L(0)$ and
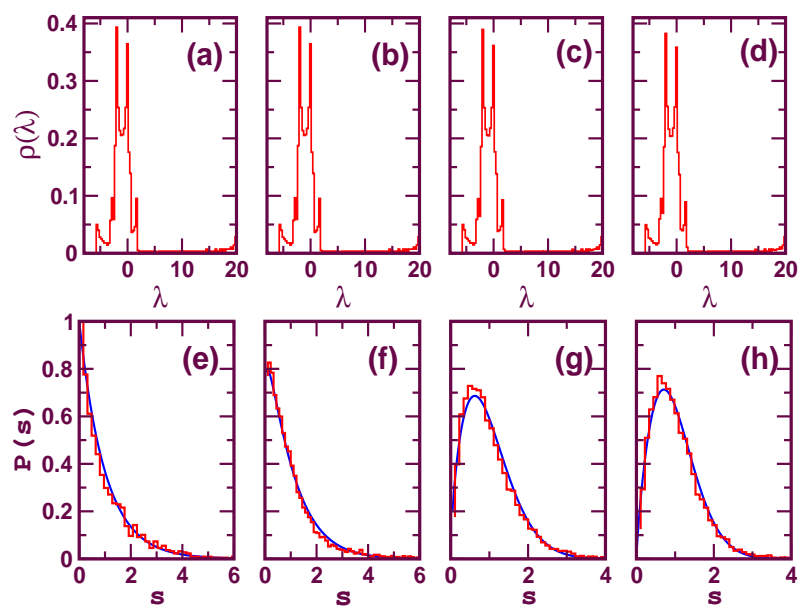

FIG. 3: (Color online) Figure shows the transition from ring regular lattice to the small-world network. (a)-(d) show the spectral densities and (e)-(h) show the corresponding spacing distributions for $p=0,5 \times 10^{-5}, 2 \times 10^{-4}, 5 \times 10^{-4}$, respectively. The histograms are numerical data and the solid lines are the corresponding fitted Brody distribution (Eq. 1). See text for the corresponding values of Brody parameters. All the networks have $N=2000$ nodes and $k=40$ average degree per node, and data are average over 10 random realization of the rewiring process. 


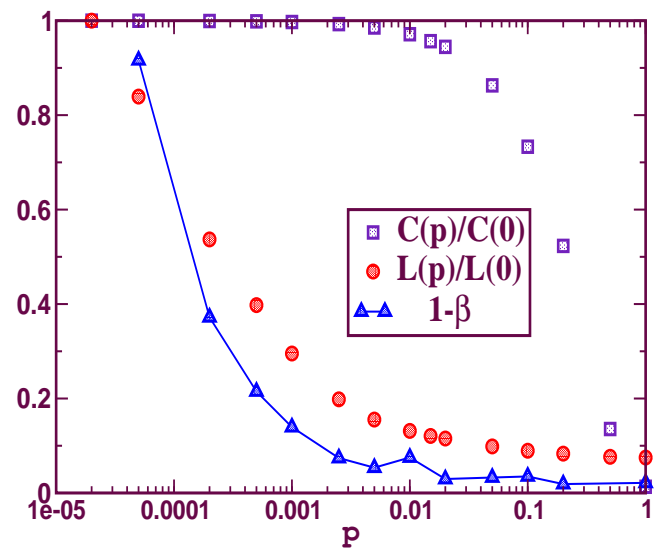

FIG. 4: (Color online) The shifted Brody parameter $1-\beta$ $(\triangle)$ is compared with the two well-known network parameters, normalized characteristic length $L(p) / L(0)(\bigcirc)$ and normalized clustering coefficients $C(p) / C(0)(\square)$. The data points corresponding to the curve for $\beta$ is joined by a solid line for better visibility. Network parameters are same as for the Fig. 3. The data are average over 10 random realizations of rewiring process for each value of $p$.

$C(0)$ for the regular lattice. Due to this normalization, at $p=0$, normalized $L$ and $C$ both are one; whereas for $p \rightarrow 1$, both network parameters will be closer to zero. However, $\beta$ behaves completely opposite way at the two extreme values of $p$. Therefore, in Fig. 4. we compare $1-\beta$ with normalized the $L$ and $C$. This figure shows that the $\beta$ and the normalized characteristic length $L(p) / L(0)$ display similar trends and strong correspondence. The most important result of this study is that the Poisson $\rightarrow$ GOE transition and the small-world transition take place at the same rewiring probability $p$. Note that all results presented here are for the adjacency matrices, however we have done similar analysis for Laplacian matrices also and for Fig. 3 and Fig. 4 qualitatively same results are obtained [17].

In summary, we study eigenvalues spacing distributions of various model networks and a real-world network. We show that though the spectral densities of the random, the scale-free and the small-world networks are different, their eigenvalues spacing distributions are same and follow GOE statistics. We also show that spacing distribution for a protein-protein interaction network in budding yeast follows GOE statistics. Following interpretation of RMT this universal GOE statistics implies that the eigenvalues are strongly correlated among themselves because of some kind of randomness in the corresponding matrix. In network concept this can be considered as sufficient amount of randomness or disorder in network connections. Furthermore, we study effect of randomness in network architecture on the eigenvalues fluctuations, and use Brody parameter to quantify this randomness. We show that there exists one to one correlation between the network diameter, which is a structural property, and the Brody parameter characterizing a spectral property. We observe that GOE transition occurs at the onset of small-world transition. Again, this result implies that at the onset of small-world transition, there is some kind of randomness spreading over the whole network leading to the strong correlations among eigenvalues. The interesting point here is that a very small amount of random connections is sufficient to give rise these correlations.

Now we point out some of the future prospects of our results. Universal GOE behavior of network spectra suggests that statistics of the bulk of eigenvalues of these networks are consistent with those of a real symmetric random matrix with entries being Gaussian distributed random numbers, and deviation from this could be understood as system specific part. Random matrix analysis of eigenvectors had been performed for various different systems [12], such as stock-market, atmosphere, human EEG to extract system specific features by separating out universal properties from time-series of these systems. In same spirit, one can consider eigenvector analysis of adjacency matrices to understand system specific features in different classes of networks [16]. The system specific features could be important nodes, links or anything; but the most important outcome of results presented in this paper is that we can apply RMT, a very well developed branch of Physics, to study networks, providing a completely new framework to the complex network research.

\section{Acknowledgments}

We thank Professor Steven Tomsovic for useful discussions and Dr. Luis G. Morelli (MPIPKS, Dresden) for useful suggestions.
[1] R. Albert and A.-L. Barabási, Rev. Mod. Phys. 74, 47 (2002) and references therein; S. Boccaletti et al., Phys. Rep. 424, 175 (2006).

[2] D. J. Watts and S. H. Strogatz, Nature 393, 440 (1998).

[3] A.-L. Barabási and R. Albert, Science 286, 509 (1999).

[4] E. Ravsaz et al., Science 297, 1551 (2002); R. Guimerá and L. A. N. Amaral, Nature 433, 895 (2005).

[5] D. M. Cvetković, M. Doob and H. Sachs, Spectra of Graphs : theory and applications, (Academic Press, 3rd
Revised edition, 1997).

[6] I. J. Farkas et al., Phys. Rev. E 64, 026704, (2001).

[7] M. L. Mehta, Random Matrices, 2nd ed. (Academic Press, New York, 1991).

[8] S. N. Dorogovtsev et al., Phys. Rev. E 68, 046109 (2003).

[9] M. A. M. de Aguiar and Y. Bar-Yam, Phys. Rev. E 71, 016106 (2005).

[10] F. Luo et al., Phys. Rev. E 73, 031924 (2006).

[11] T. Guhr et al., Phys. Rep. 299, 189 (1998). 
[12] For examples, V. Pleron et al., Phys. Rev. Lett. 83, 1471 (1999); M. S. Santhanam and P. K. Patra, Phys. Rev. E 64, 016102 (2001); P. Seba, Phys. Rev. Lett. 91, 198104 (2003).

[13] T. A. Brody, Lett. Nuovo Cimento 7, 482 (1973).

[14] http://vlado.fmf.uni-lj.si/pub/networks/data/bio /Yeast/Yeast.htm.
[15] S. Jalan and J. N. Bandyopadhyay, e-print : cond-mat/0701043.

[16] S. Jalan, J. N. Bandyopadhyay and M. S. Santhanam (under preparation).

[17] S. Jalan and J. N. Bandyopadhyay, e-print : cond-mat/0611735 